\title{
An Evolution of Proxy Mobile IPv6 to the Cloud
}

\author{
Ákos Leiter \\ Nokia Bell Labs \\ Budapest, Hungary \\ akos.leiter@nokia-bell-labs.com
}

\author{
Nándor Galambosi \\ Nokia Bell Labs \\ Budapest, Hungary \\ nandor.galambosi@nokia-bell- \\ labs.com
}

\author{
László Bokor \\ Department of Networked \\ Systems and Services, Faculty of \\ Electrical Engineering and \\ Informatics, Budapest University \\ of Technology and Economics \\ Budapest, Hungary \\ bokorl@hit.bme.hu
}

\begin{abstract}
Network Function Virtualization (NFV) and Software Defined Networks (SDN) do not leave any legacy network services untouched. This work will present how Proxy Mobile IPv6 (PMIPv6) can evolve to the cloud. Our approach is to introduce this evolution within a step-by-step architecture guideline while keeping standards compatibility. We also show how PMIPv6 can fit into a central cloud - edge cloud environment on the top of Kubernetes and Openstack under a unified orchestration umbrella. The proper integration of PMIPv6 into this new environment does not just enforce acquiring new capabilities, e.g., scaling PMIPv6 elements; it also can ensure closed-loop orchestration where PMIPv6 can be controlled continuously by the actual network needs.
\end{abstract}

\section{CCS CONCEPTS}

Networks -> Network Architectures

\section{KEYWORDS}

PMIPv6; Kubernetes; Openstack; ONAP; cloud-native; CNPMIPv6

\section{ACM Reference format:}

Ákos Leiter, Nándor Galambosi and László Bokor 2021. An Evolution of Proxy Mobile IPv6 to the cloud. In Proceedings of $19^{\text {th }}$ ACM International Symposium on Mobility Management and Wireless Access (MobiWac'21), Alicante, Spain. November 22-26, 2021, ACM NY, NY, USA. 9 pages. https://doi.org/10.1145/3479241.3486684

\section{Introduction}

Proxy Mobile IPv6 (PMIPv6) [1] is an operator-centric protocol designed to handle Mobile Nodes' (MN) mobility at the IP level. It

\footnotetext{
Permission to make digital or hard copies of all or part of this work for personal or classroom use is granted without fee provided that copies are not made or distributed for profit or commercial advantage and that copies bear this notice and the full citation on the first page. Copyrights for components of this work owned by others than ACM must be honored. Abstracting with credit is permitted. To copy otherwise, or republish, to post on servers or to redistribute to lists, requires prior specific permission and/or a fee. Request permissions from Permissions@acm.org.

MobiWac '21, November 22-26, 2021, Aliante, Spain

(C) 2021 Association for Computing Machinery.

ACM ISBN 978-1-4503-9079-8/21/11 ..\$15.00

https://doi.org/10.1145/3479241.3486684
}

is a promising technology, e.g., to support vertical handovers in heterogeneous networks [2] [3] [4] [4]; however, its deployment penetration has not yet reached critical volume. Recent trends and technological advances could alter this thanks to the severe changes in the IT industry and telecommunication (cloud, microservices, NFV, SDN, etc.) that seriously affected the environment where and how a network service must run [5] [6] [7]. This paper will discuss how PMIPv6 can fulfill the new requirements and evolve to the new era that could push the protocol towards real-life deployments. We provide a step-by-step evolutionary approach to how a legacy PMIPv6 implementation and environment changes to the cloud. We also consider Multiaccess Edge Computing (MEC) [8] - whose goal is to move services as close as possible to end-users - and analyze how edge clouds must be involved in the cloudification of PMIPv6. Furthermore, services in clouds are orchestrated, which also cannot be avoided by the new design of PMIPv6. PMIPv6 must comprise new interfaces designed for orchestration procedures. Openstack [9] and Kubernetes [10] are our primary target environments during the architecture review of PMIPv6; however, virtual machines (VM) and containers are general enough to make them run on other platforms like VMware [11] as private or Google Cloud [12] as public cloud solutions. We present the High-level Design (HLD) of such a PMIPv6 scheme called Cloud-Native PMIPv6 (CN-PMIPv6), and we also address the packet flows at Low-level Design (LLD). Furthermore, there are measurements to conclude containerization overhead for PMIPv6type traffic.

Our work keeps in mind that the newly introduced CN-PMIPv6 must be compatible with IP-based mobility management's existing standards while moving the whole network service into the cloud. We admit that this paper is just the beginning and only shows a possible PMIPv6 cloud evolution. We hope this architecture proposal contributes to the "final" architecture of a novel and deployment-friendly PMIPv6 solution.

The remaining sections are organized as follows: Section 2 is about related works. The architectural context is shown in Section 3. An evolutional path of PMIPv6 is detailed in Section 4, while the high-level design of CN-PMIPv6 is elaborated in Section 5. The Control plane flow of CN-PMIPv6 is depicted in Section 6. Low- 
level design is in Section 7. Proof of Concept testbed and measurement results are demonstrated in Section 8. Conclusion and future work are placed at Section 9 and 10, respectively.

\section{Related Works}

To the best of our knowledge, NFV and cloud-native aspects of PMIPv6 were not examined so far; however, some related work deals with SDN and PMIPv6 concepts. Furthermore, this paper is the natural continuation of Cloud-Native Mobile IPv6, published earlier by a subset of this paper's authors[13]. There are several requirement emerged for making IP-based mobility cloud-native, which can be found in [13].

$S$ Kim et al. [14] proposes PMIPv6 integration in SDN environments. They use Openflow, and their goal is to eliminate tunneling overhead while keeping standard PMIPv6 signaling (OPMIPv6). Syed M Raza et al. [15] keeps tunneling and standardized PMIPv6 control messages while the underlying network and SDN-controlled with Openflow (OF-PMIPv6). Kyoung-Hee Lee [16] also eliminates the tunneling overhead of PMIPv6, but Routeflow and Openflow are used. You Wang et al.'s concept [17] does not maintain standard PMIPv6 control messages, and Openflow solves mobility management. Tunneling is also eliminated. Sakshi Chourasia et al. [18] examine PMIPv6 and SDN connection in Evolved Packet Core (EPC). Control messages of standard PMIPv6 are not used, and with the help of the SDN concept, they eliminate tunnel usage.

\section{The Context of Cloud-native Proxy Mobile IPv6}

\section{A. Evolution to containers with Orchestration}

One of the consequences of NFV is to separate network software from hardware. Thus, the software does not need to be built on specialized hardware kits. Furthermore, software modules can also be decoupled and can be handled independently. One of the ways of software separation is to put them into individual VMs. The next evolution step is to put software into containers that only consist of the minimal dependencies of software and do not run a new kernel instance like VMs.

The more VMs and containers the system has, the more complex resource management is needed. This leads to the orchestration of VMs and containers. Several (private) cloud orchestrators, e.g., Openstack, VMware which can support telco clouds. For container orchestration, we only deal with Kubernetes. The atomic orchestration element of Kubernetes is a POD that consists of multiple Docker containers. Figure 1 depicts the runtime context of a cloud-native telecommunication service. In this case, VMs are Kubernetes Worker nodes. Of course, it is possible to eliminate VMs at all by having bare-metal Worker nodes. Still, the containers can add no new kernel modules, just using the kernel modules of blades: thus, Worker nodes can lose their generality. Of course, the best is when kernel modules are libraries inside containers. Pods are coupled with Kubernetes Services [19] which are the constant entry points of POD-enabled functionalities due to POD mortality. Kubernetes PODs and Services are organized into Kubernetes Deployments[20] whose primary benefit is selfhealing on POD level. If a POD's health check is not OK, Kubernetes redeploys it to mitigate service malfunctions automatically. This is the environment where a cloud-native PMIPv6 should be aligned to.

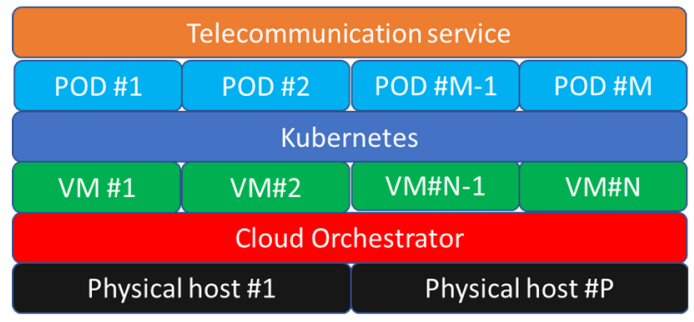

Figure 1 - Cloud and container-based environment for telecommunication services

\section{B ETSI Management and Orchestration Framework (ETSI MANO)}

ETSI has standardized a framework where Virtual Network Functions (VNFs) can run. This is the ETSI NFV MANO framework[21], presented at Figure 2

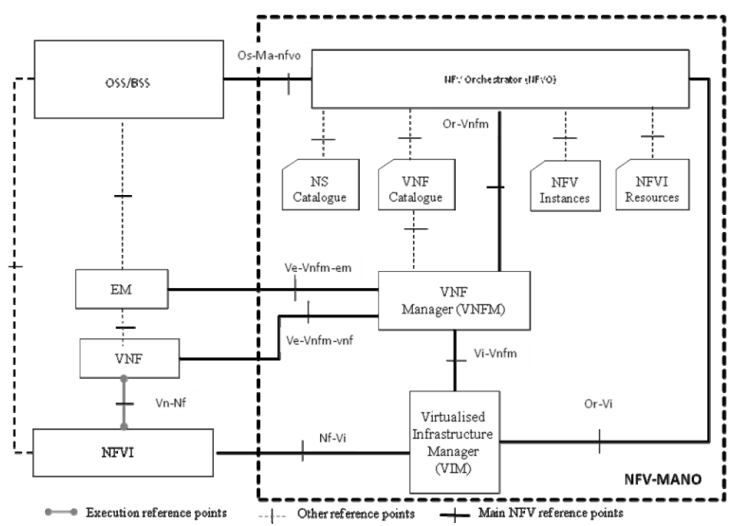

Figure 2 - ETSI NFV MANO Architecture[21]

It defines how network services should be managed in a virtualized telecommunication environment. The essential elements of ETSI MANO:

- NFV Orchestrator (NFVO): central network service orchestrator

- VNF Manager (VNFM): responsible for life-cycle management of $\mathrm{VNF}$

- Virtual Infrastructure Manager (VIM): in our case, it's Openstack or Kubernetes

These have well-defined interfaces too, which are requirements for telecommunication services interoperability. Network Service Descriptor (NSD) and VNF Descriptor (VNFD) are responsible for defining elements declaratively. When writing this paper, ETSI standards do not differentiate VNF and Container/Cloud-Native Network Functions (CNF), so we regard Kubernetes-based services as another VIM zone. However, we think there will be 
differences in CNF as at least typical orchestration is needed for Openstack and Kubernetes, depicted in Figure 1, to run a CNF. Furthermore, Open Network Automation Platform (ONAP) [22], which deals with ETSI NFM MANO implementations, too, has proposals for CNF-ready orchestration at [23]. Still, this discussion is out of the scope of this paper.

\section{C Edge Clouds}

Edge clouds are placed as close as possible to end-user to support several use-cases, e.g., low-latency communications, localbreakout. Edge cloud deployments can host VNFs that run on provider/customer edge devices. In our CN-PMIPv6 use-cases, edge cloud is an excellent candidate to host MAG nodes because MAGs are typically close to MNs. There are dedicated edge cloud solutions for orchestration Kubernetes lightweight version, K3S[24] and Openstack also deals with the edge. It is essential to be as lightweight as possible because of the high cost of deployments of edge clouds[25] [26] [27] . Furthermore, edge clouds give a new layer of orchestration when distributed applications like CN-PMIPv6 LMA and MAG run in separate data centers, but initial deployments can be simultaneous.

\section{D Use cases of CN-PMIPv6}

This Section presents a few use-cases where PMIPv6 can be applied and needs our proposed cloud-transformation. The environment of these use-cases is being changed from traditional, physical device-oriented architectures to cloud-native.

\section{D.1 PMIPv6-based edge offload}

While MIPv6 is rather an enterprise-grade service, PMIPv6 is optimized for Service Provider (SP) usage. PMIPv6 MAG could be located on the edge cloud where traffic can be offloaded to avoid reaching the core network and increasing the load. This is where the scalability of a cloud-native PMIPv6 can come into the picture. MAG capacity can be scaled-in, and other edge services can consume the freed-up capacity. This is crucial as edge cloud deployments have a considerable cost impact[25] [26] [27] .

\section{D.2 Service Provider Wi-Fi with PMIPv6}

PMIPv6 is a suitable protocol to connect non-3GPP access to $4 \mathrm{G} / 5 \mathrm{G}$ networks via S2 interface family [28]. This is the base of a kind of Service Provider Wi-Fi solution. With the introduction of CN-PMIPv6, it can be scalable and adjusted to the current traffic needs to save resources. On the edge with MAG, scaling can even be more resource friendly.

\section{Architectural evolution to Cloud-native Proxy Mobile IPv6}

We can see a trend where every layer of the network gets computational functionalities. The most straightforward example is the edge cloud, whose primary purpose is to be as close as possible to the end-user to enhance communication latency. Those functions like PMIPv6 MAG close to end-users can be decoupled and placed in an edge cloud datacenter. With the advent of SDN, it is possible to lead corresponding IPv6 control traffic to the nearest MAG. Furthermore, the core network is also being virtualized (central cloud) where PMIPv6 LMA runs and brings in orchestration functionalities and layers. With these new service placement possibilities, it is worth examining a possible evolution of PMIPv6 to the cloud in a step-by-step manner.

\section{A Standard PMIPv6}

Ordinary PMIPv6 LMA and MAG is a software component of a router. Usually, LMA is placed to a central router or 3GPP PGW, which serves 3GPP S2 interface family [28]. MAGs can run on edge router or Wireless LAN Controllers (WLC). PMIPv6 is also a tunneling protocol on 3GPP S5 interface, where it coexists with GPRS Tunneling Protocol (GTP) [29]. The latter usage is not examined in this paper. Figure 3 represents the manner how PMIPv6 can be used to connect non-3GPP networks to EPC. Every software component of PMIPv6 can be implemented as a dedicated router software.

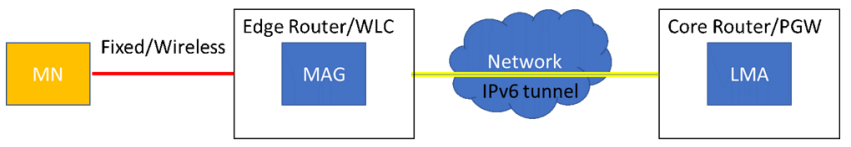

Figure 3 - Standard PMIPv6 architecture

\section{B Virtual machine-based PMIPv6 LMA}

The next step of the evolution is when LMA is decoupled from being a router software. It runs now inside a VM on Commercial off-the-shelf (COTS) hardware (Figure 4). This is a consequence of NFV. MAG remains as it is.

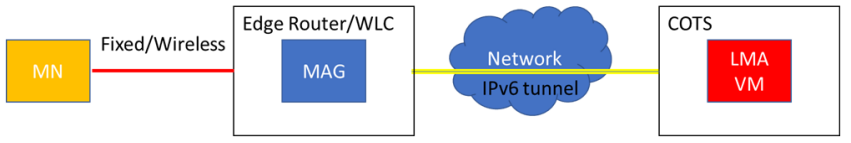

Figure 4 - PMIPv6 LMA in a virtual machine

\section{C Virtual machine-based LMA with Binding Cache separation}

As LMA is pulled out from routers, it is now possible to separate LMA functions: Binding Cache (BC) is a database, no need to be integrated inside LMA: BC can be a separate VM (Figure 5) that works independently.

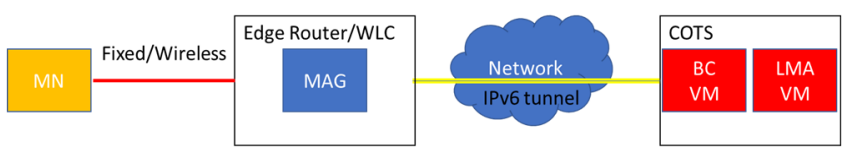

Figure 5 - PMIPv6 LMA in VM with BC separation

\section{D PMIPv6 LMA with Virtual Infrastructure Manager}

As BC is a database and VIMs have storage services (Openstack Cinder [30], Openstack Swift [31] there is no need to handle BC operation combined with LMA (Figure 6). Now LMA administrators do not need to do anything with $\mathrm{BC}$ reliability; the infrastructure solves it. If Cinder is responsible for giving 
persistent data storage for database VM, data loss may be avoided in case of database process failures. LMA VMs are orchestrated at this stage too, and, e.g., HEAT templates [32] are used to describe them. If LMA VM is clustered, then VIM can scale it even.

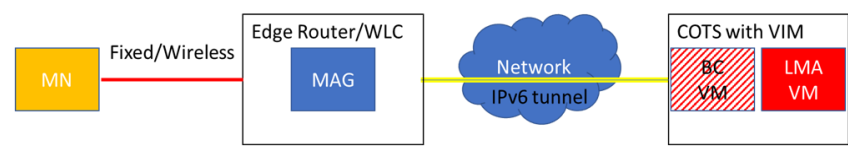

Figure 6 - PMIPv6 LMA with VIM zone

\section{E PMIPv6 LMA as a Network Service in ETSI MANO context}

When it comes to telecommunication environment and service standardization, this is the step to have it. At this point, LMA becomes a VNF, particularly, life-cycle managed by a VNFM and orchestrated by an NFVO. Also, standardized NSD and VNFD describe its way of working by TOSCA[33] . LMA is transferred to be a network service (Figure 7).

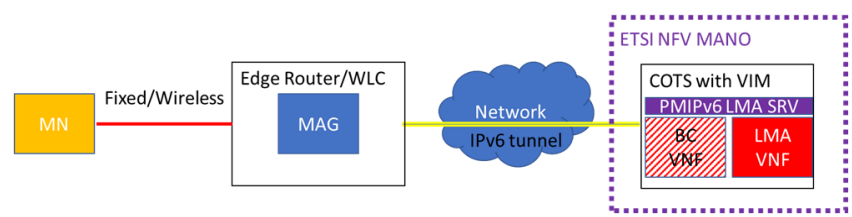

Figure 7 - PMIPv6 LMA with ETSI MANO

\section{F Container-based PMIPv6 LMA with Virtual Infrastructure Manager}

This is one step back from Section 4.E as it is without ETSI MANO. But VMs are changed to containers, presented in Figure 8 . Here, LMA software is coupled together into a container, and they can even be clustered containers. Also, these can be bare-metal without VMs, but containers will depend on the underlying kernel modules; it is not possible to add new kernel modules by containers.

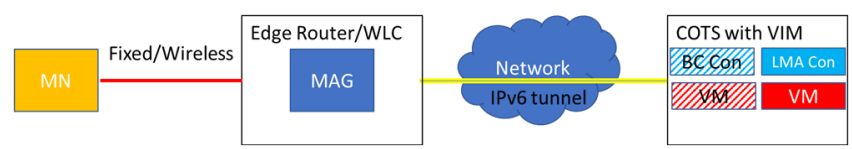

Figure 8 - Container-based PMIPv6 LMA

\section{G PMIPv6 LMA in containers with Kubernetes}

Containers can be orchestrated, too, independently from VM orchestration. At this phase, Kubernetes also couples containers together to PODs, then Kubernetes Services [19] (Figure 9). Scaling can be possible to increase the number of PODs behind a particular Service. Furthermore, Kubernetes Deployment [20] introduces new fault-tolerance solutions as it examines PODs's health continuously and almost immediately redeploys them if failure is detected.

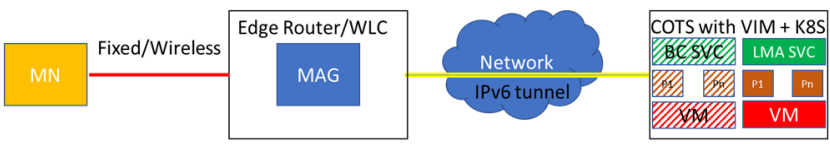

Figure 9 - PMIPv6 LMA on the top of Kubernetes

\section{H Carrier-Grade PMIPv6 LMA}

There are several network domains like core, data center, and transport (Figure 10). Traffic should go through these undisturbedly and reach the processing place no matter if it's a container or a VM. But VM and container orchestrations put two more layers on top of the existing ones. SDN controllers can help to overcome this complexity increase of the multiple overlay network layers. MAG edge router can be SDN compatible, too, to be controlled by a transport or access SDN controller. To detail these scenarios is out of the paper's scope because of the considerable complexity: it could quickly fill a separate article.

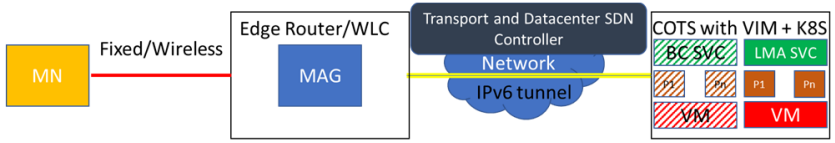

Figure 10 - Carrier-grade PMIPv6 LMA

\section{I PMIPv6 MAG on the edge}

Now let's move on to virtualize and containerize MAG too! There are a cloud and a container orchestrator at the edge cloud segment as well, and on top of them, MAG can run inside PODs, as depicted in Figure 11. The same evolutional steps can be applied here, just like at the LMA part. Now every component of PMIPv6 can be regarded as cloud-native. Bare-metal containers are applicable choices with their mentioned drawbacks in Section 3.A

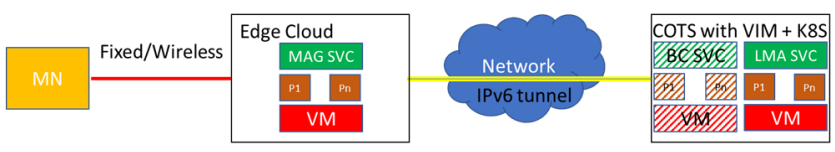

Figure 11 - PMIPv6 MAG on the edge

\section{J Inter-domain orchestration of Cloud-native PMIPv6 LMA and MAG}

Orchestration should not be done separately at central and edge clouds. The final step is when every component of CN-PMIPv6 is controlled together under a unified orchestrator (Figure 12). Furthermore, based on actual traffic needs, MAG should not have to be placed everywhere; its placement can be optimized. E.g., ONAP Optimization Framework (OOF) [34] can deal with application placement based on specific applications' needs. It is worth examining of CN-PMIPv6 MAG placement strategies, mainly detecting inter-MAG handover intent (based on predictions) and prepare the corresponding edge cloud to start MAG service itself (this is again out of the scope of this paper). Furthermore, Kubernetes Federation [35] can be one way to have an infrastructure-level unified container orchestration. 


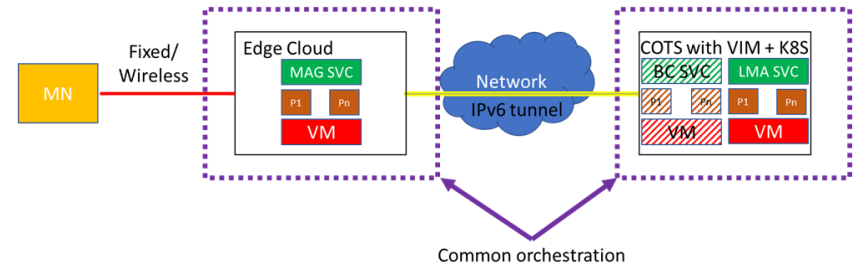

Figure 12 - Unified orchestration of Central and Edge clouds for CN-PMIPv6

\section{High-level design of CN-PMIPv6}

Remarkably, in the case of PMIPv6, Home Network Prefix (HNP) is moved by LMA and MAG at the edge of the PMIPv6 domain. Every Corresponding Node $(\mathrm{CN})$, which represents anything outside the PMIPv6 domain, knows only that: route every traffic to the LMA which is directed to an MN. LMA advertises MNrelated routes. When an MN attaches to the PMIPv6 domain, aka to a link whose L3 is maintained by a MAG, MAG examines if MN is allowed to join at all. If yes, then it registers it at the LMA (Proxy Binding Update (PBU)/Proxy Binding Acknowledgment (PBA)). LMA sends the HNP to the MAG; then MAG selects an IPv6 address from HNP, and MAG adds that IPv6 address to its interface, directed to MNs. This means multiple IPv6 addresses are on one interface.

With Router Advertisement (RA), MAG advertises the HNP to the $\mathrm{MN}$. MN has multiple choices to generate an address (autoconfiguration or DHCP, etc.). But the generated address should be the same as it used to be. Thus, L3 stays unbroken. Maybe, the received address at the previous MAG is still valid, so basically, it is just an extension of IPv6 address validity. As HNP is moved to a new link, LMA should adjust its routing to this new IPv6 domain reachability: LMA will set the HNP as reachable via another tunnel. This is how PMIPv6 hides MNs' movements from the outside world: there is no need to know where a $\mathrm{MN}$ is, as LMA keeps maintaining routing with tunnels and only advertises that MNs with their HoA are reachable via it.

Figure 13 shows the IPv6 prefix movements' way of operation. However, it does not require to dedicate a gateway address for every home link emulation between MAGs and MNs if all the MAG uses the same link-local address, and MNs can use this as the default route. But this leads to a security consideration: even though MN has a valid address from its HNP, it cannot forward packets on that IPv6 address until MAG does not setup up a gateway address from HNP of the particular MN. This is also protection: if $\mathrm{MN}$ somehow acquired a valid HNP IPv6 address, it cannot use it to present itself until this "IP island" is not connected via MAG. Also, this process assumes end-to-end L2 connectivity between MAGs and MNs.

By default, a shared tunnel between LMA and MAG is enough for multiple MNs. But MNs may need individual tunnel properties: this leads to Multiple LMA Addresses (MLMAA) at LMAs and Multiple Proxy Care-of-Addresses (MPCoA) at MAGs. At first glance, MLMAA should be at the same IPv6 prefix, to be advertised outside by routing protocols aggregately. The LMA's responsibility is to handle which LMAA belongs to which
MAGs/MNs. When a packet arrives at the LMA, it needs to choose the right tunnel and the right source LMAA for the corresponding MN. At BC, LMA must maintain the connection of LMAA, PCoA, and HNP, and based on this database, LMA must continuously adjust its (policy) routing.

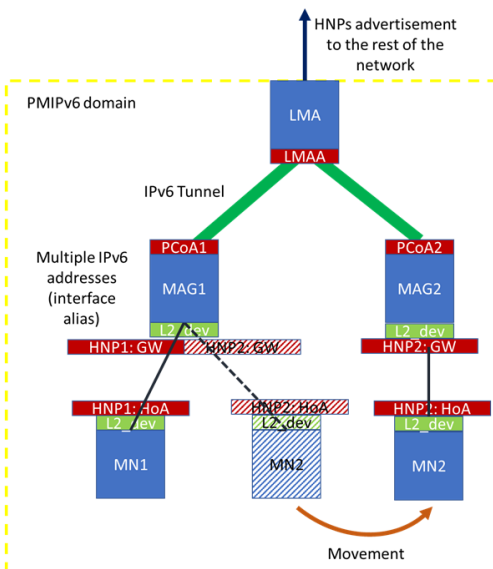

Figure 13 - Overview of original PMIPv6 IPv6 address management with an inter-MAG handover

The control plane (handling PBU/PBA) is separated from the data plane where tunneled traffic goes. Thus, in our proposal of $\mathrm{CN}-$ PMIPv6, LMA and MAG have two types of services: Control Plane Service (CPS) and Data Plane Service (DPS). DPS is divided into Generic Data Plane Service (G-DPS) and Specialized Data Plane Service (S-DPS). G-DPS and S-DPSs on LMA hold LMAAs of MNs. If a $\mathrm{MN}$ does not require special functions, like flow mobility, an IPv6 address is added from MN HNP to the MAG G-DPS and the corresponding LMAA to LMA-G-DPS (if an MN is allowed to join to the PMIPv6 domain). The PODs of LMA and MAG G-DPS are responsible for terminating tunnels of $\mathrm{MNs}$. If a $\mathrm{MN}$ requires flow mobility, then a new S-DPS is created and addressed by an LMAA where rules can be applied to handle flow mobility. Flows are detected on S-DPS by 5-tuple, and flows are forwarded to a dedicated POD. Thus, every flow can be processed independently by individual PODs, ensuring flow-based policies (e.g., individual charging by traffic types). But there can be two layers for S-DPS: if a flow has outstanding traffic, then one POD may not be enough to process traffic: this is where the second layer of S-DPS (L2-SDPS) comes into the picture. L2-S-DPS can couple together several PODs to load balance traffic connected to a particular flow. But this should not be mandatory by default. Furthermore, this is the topic of service meshes, but service mesh examination is out of this paper's scope. MAGs follow the same design principles, but IP addresses are allocated from HNP or PCoA pool. Figure 14 presents the high-level design of CN-PMIPv6. The yellow line is the control channel; the dotted lines represent the data path. The red dotted line depicts the not tunneled traffic from MNs to MAGs, while the green dotted lines show the IPv6 tunnel traffic. In the case of flow mobility, it is possible to have LMA S-DPS PODs, but not having MAG S-DPS PODs, e.g., there may be certain policies, like traffic aggregation, which do not need S-DPS on MAGs. 


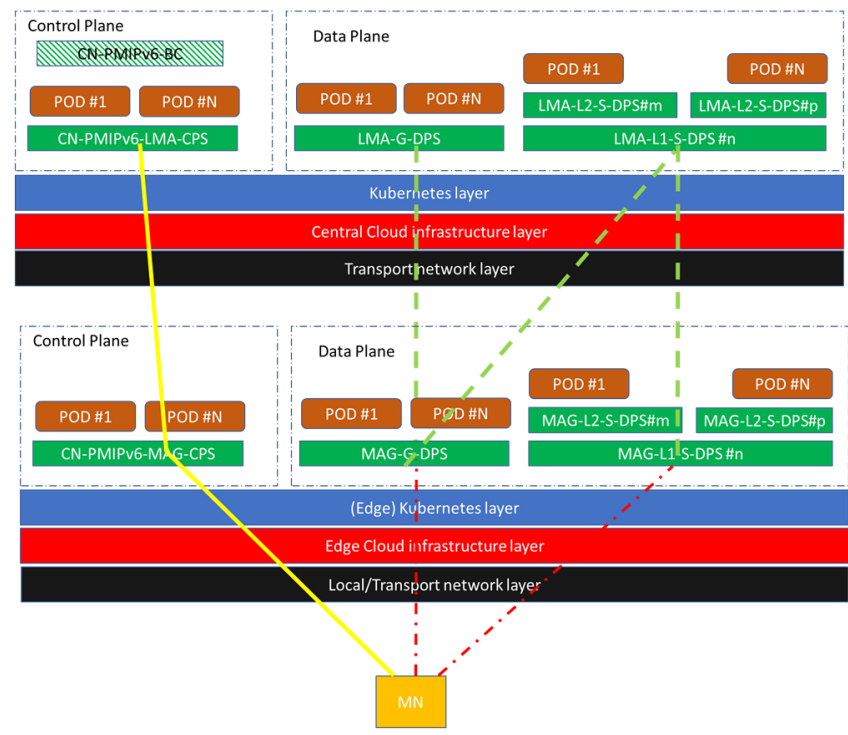

Figure 14 - High-level design of CN-PMIPv6 two-layer architecture

However, for local traffic breakout of a specific flow on MAG can be done via only MAG S-DPS for a particular flow of MN. One thing to highlight: at (CN)PMIPv6 flow mobility, MNs should have modifications at least for receiving flow separation commands, and MNs have to be able to execute them by flow separation.

\begin{tabular}{|c|c|c|c|c|}
\hline & \multirow{2}{*}{$\begin{array}{c}\text { Without Flow } \\
\text { mobility }\end{array}$} & \multicolumn{3}{|c|}{ With Flow mobility } \\
\hline & & $\begin{array}{c}\text { LMA- } \\
\text { support }\end{array}$ & $\begin{array}{c}\text { MAG- } \\
\text { support }\end{array}$ & Both \\
\hline $\begin{array}{l}\text { LMA } \\
\text { G-DPS }\end{array}$ & OK & N/A & $\mathrm{OK}$ & $\mathrm{N} / \mathrm{A}$ \\
\hline $\begin{array}{l}\text { LMA } \\
\text { S-DPS }\end{array}$ & N/A & OK & $\mathrm{N} / \mathrm{A}$ & $\mathrm{OK}$ \\
\hline $\begin{array}{l}\text { MAG } \\
\text { G-DPS }\end{array}$ & OK & OK & $\mathrm{N} / \mathrm{A}$ & N/A \\
\hline $\begin{array}{l}\text { MAG } \\
\text { S-DPS }\end{array}$ & N/A & N/A & $\mathrm{OK}$ & OK \\
\hline
\end{tabular}

Table 1. Table summary of DPS by mobility cases

Table 1 summarizes which DPS should run in case of certain mobility scenarios. Bidirectional tunnels are set up between PCoA and LMAAs. Every LMA DPS POD has its own service address to have a unique identification. Therefore, multiple LMAA exits. So, routing to the outside world should advertise that on which LMAA is reachable a certain HNP. This means every LMA DPS should run a routing protocol. This is one reason why PMIPv6 domain exists because PMIPv6 domain hides internal routing and network setup from the outside world for the corresponding mobility events.

\section{Control Flow}

There are two types of Binding Registration in the case of $\mathrm{CN}-$ PMIPv6:

- $\quad \mathrm{MN}$ does not require flow mobility (Figure 15)

- $\quad$ MN requires flow mobility (Figure 17)
In the first case (without flow mobility, Figure 15), MN's traffic is assigned to the MAG-G-DPS and LMA-G-DPS: new IP addresses are assigned to handle that specific traffic of the newly registered $M N$. From MN point of view, the control flow is similar to PMIPv6 standards: Router Solicitation (RS) and Router Advertisement (RA) ICMPv6 messages are used for communicating with MAG. When MN attaches to the network, it sends RS, which the MAGCPS detects. MAG-CPS then sends PBU to the LMA CPS, which then updates BC. Then LMA CPS updates the LMA-G-DPS with the new IPv6 address. Now LMA-G-DPS can advertise that to the outside world, the HNP is reachable via it. Notably, a new LMAA is assigned to the $\mathrm{MN}$, and $\mathrm{HNP}$ is advertised to be reachable via that LMAA. After that, LMA CPS sends a PBA with the HNP to MAG CPS. MAG CPS chooses an address from the HNP (advised to be the first in the domain) and adds that IPv6 address for the MAG-G-DPS service which will be the default route for the MN. From this point, it is possible to use the bidirectional IPv6-in-IPv6 tunnel between LMA-G-DPS and MAG-G-DPS. MAG CPS sends the RA to the MN, including the HNP. Then MN can setup its HoA (which is generated by the EUI64 algorithm using HNP) and start using the link.

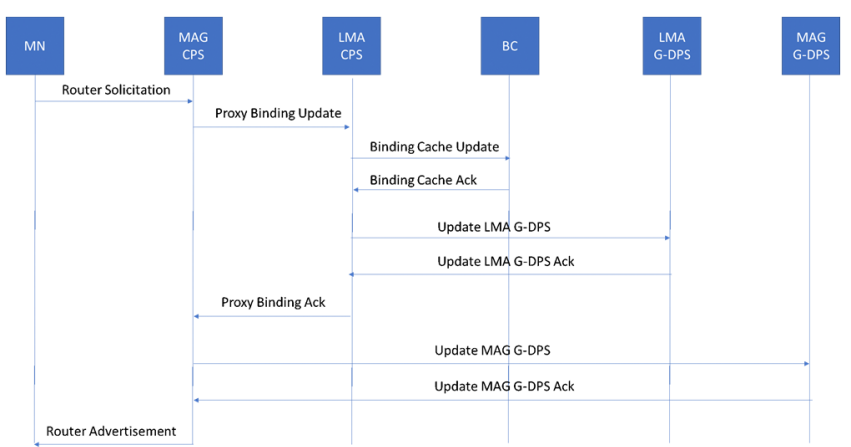

Figure 15 - CN-PMIPv6 Binding Registration without Flow Mobility

The second case, when flow mobility is required, is depicted in Figure 17. Here, at the LMA side, a new LMA-S-DPS service is created, dedicated for that particular MN. There are two subcases for CN-PMIPv6 flow mobility: 1) MAG-G-DPS is used: there is no dedicated service for MN at MAG; everything is tunneled through MAG-G-DPS. 2) MAG-S-DPS is used: new MAG-S-DPS is created for that particular MN.

At the end of the registration with flow mobility with PBA, the MAG can set up the new MAG-S-DPS and sends the RA with HNP to the MN. So, MAG-S-DPS can separate flows and can handle flows by policy at its PODS. Thus, there is no need for end-user modification. But there can be a further step when flows are separated on MN, too: this is where Flow Policy Update and Ack can come into the picture [36]. This may be needed for traffic offload purposes, for example, if $\mathrm{MN}$ has multiple interfaces and LMA decides where to route certain flows on particular interfaces. Of course, this approach requires end-user software extension. According to the current network needs, flow Policy Update can be dynamic, driven by LMA. Predefined flow rules may be 
available too. Examining flow rules assignment is out of the scope of this paper.

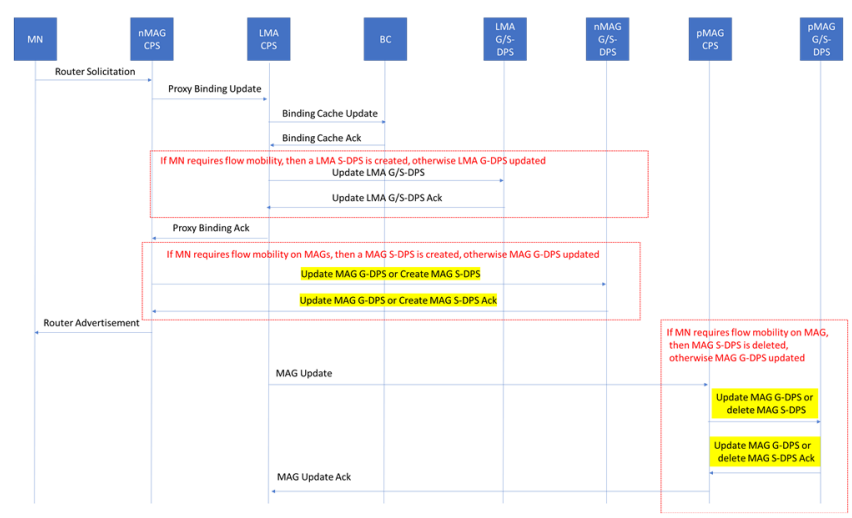

Figure 16 - CN-PMIPv6 inter-MAG handover control flow

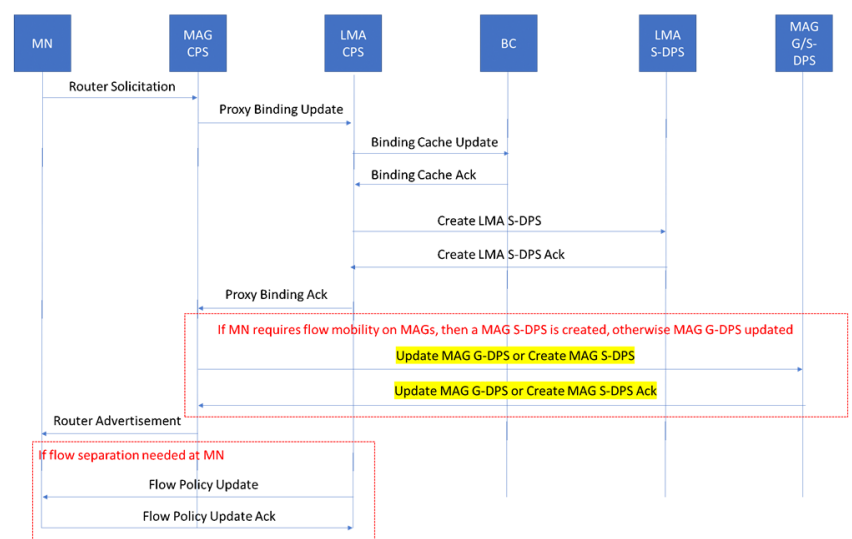

Figure 17 - CN-PMIPv6 Binding Registration with Flow Mobility

PMIPv6 deals with inter-MAG handover too where an MN changes its MAG: 1 ) it is not mandatory that, the closest router to MN should be the MAG, 2) Inter-technology handover (WiFi-4G) can also be possible. Figure 17 depicts the inter-MAG handover of CN-PMIPv6. There are two types of MAG entities: previous MAG (pMAG) and next MAG (nMAG). During an inter-MAG handover, BC should be updated, and the LMA DPS services should setup new tunnels and delete old ones to the new MAG DPS service. Furthermore, on pMAG, corresponding MAG S-DPS should be destroyed, or MAG-G-DPS should be updated because of no need for them for that MN.

\section{Low-level design of CN-PMIPv6}

With the Low-Level Design (LLD), we try to show the packet flow details inside a CN-PMIPv6 domain (Figure 18). The packet flow's purpose is "to protect" packets originated from $\mathrm{CN}$ and $\mathrm{MN}$ during packet processing: the original IPv6 packet header must be kept same no matter whether it is address translated, load-balanced, etc. This, of course, leads to setup NAT databases and maintain them. Figure 18 presents both incoming and outgoing traffic to the PMIPv6 domain. MN sends packets with its source address
(HNP\#1) and $\mathrm{CN}$ as the destination address. When packets arrive at a MAG DPS, it will change the destination address to the POD address to send packets to execution. Inside the Pod, the packet's destination address must be changed back (DNAT again) to $\mathrm{CN}$, and the packet can be tunneled now. The tunnel's source address is the POD address, and the destination address is the corresponding LMAA. When packets leave POD, their source address will be changed to PCoA. But the tunnel payload remains the same. A similar situation occurs at the LMA DPS side, and the tunnel header is eliminated. Now the original packet must be allowed to leave unchanged by the LMA DPS (no NAT). Packet receiving follows a similar logic but in the opposite direction.

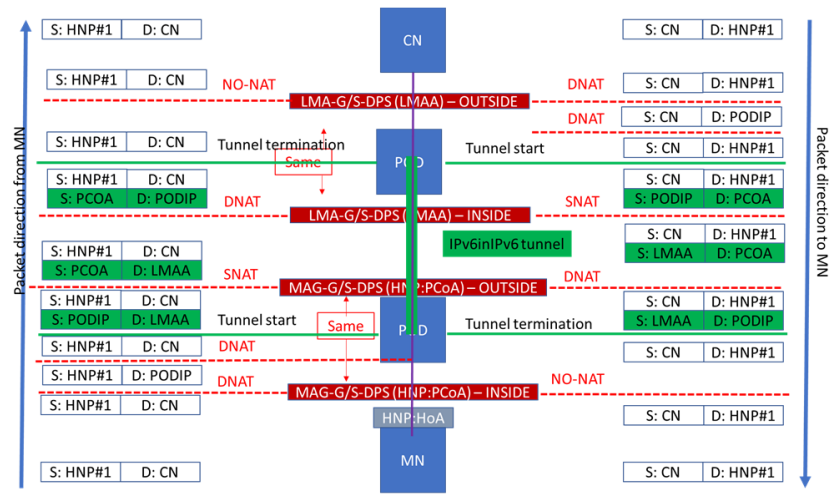

Figure 18 - Low-level design of CN-PMIPv6

\section{Measurements}

\section{A Testbed design}

Figure 19 depicts how our proof-of-concept testbed is built up to measure the characteristics of CN-PMIPv6 containerization. There is a physical node where three VM run: two separated VMs for two one-node Kubernetes cluster which are connected via a router in order to avoid direct connection. The goal is to conclude if there is any degradation of PMIPv6 control plane and user plane if the LMA and MAG runs in containerized environment compared to a native VM.

There are three measurements scenarios (Figure 19):

- VM - VM link (yellow line): LMA and MAG run inside the global namespace of a VM

- Docker container - Docker container link (blue line): LMA and MAG run inside a Docker container

- Pod to Pod link (brown line): LMA and MAG run inside a Kubernetes POD

All the links have native IPv6 connections in different subnets. Docker containers and Pods are connected via MACVLAN to the underlying VM. 


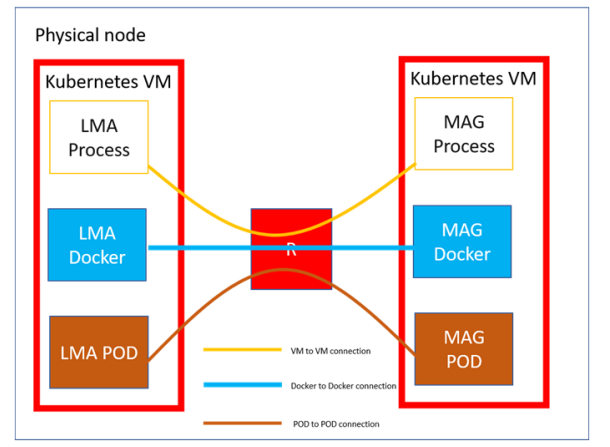

Figure 19 - Testbed for measuring containerization overhead

\section{B Control Plane measurements}

Goal: measuring the time between sending a PBU and receiving a PBA on MAG and conclude containerization overhead. Measurements have been executed 1000 times on all of the three environments (3000 times altogether).

\section{Latency between PBU and PBA on MAG in ten thousandths of a millisecond}

VM-to-VM $\square$ Docker-to-Docker

Pod-to-Pod

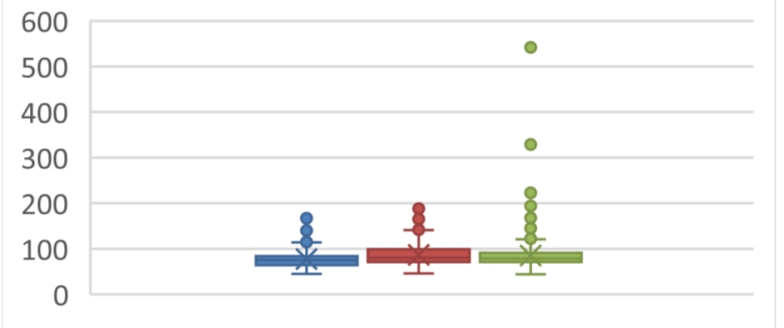

Figure 20 - Box plot of results for the time between sending PBU and receiving PBA on MAG

\begin{tabular}{|c|c|c|c|c|c|}
\hline & $\begin{array}{c}\text { Average } \\
(\mathbf{m s})\end{array}$ & STDEV & MIN & MAX & Median \\
\hline VM-VM & 7.7 & 1.8 & 4.5 & 18.6 & 7.5 \\
\hline $\begin{array}{c}\text { Docker- } \\
\text { Docker }\end{array}$ & 8.6 & 2.4 & 4.6 & 20.5 & 8.0 \\
\hline $\begin{array}{c}\text { POD- } \\
\text { POD }\end{array}$ & 8.5 & 2.9 & 4.4 & 54.2 & 7.9 \\
\hline
\end{tabular}

Table 2. - Numerical results of Control Plane measurements

Results: Docker and Kubernetes-based environment shows significant increment in average latency $(\sim 11 \%)$ as depicted at Table 2. The more "complex" the system is, the higher the standard deviation is; furthermore, the number of outliers increases as well Figure 20.

\section{C User Plane measurements}

Goal: measuring TCP throughput in IPv6inIPv6 tunnel and conclude containerization overhead between LMA and MAG. Measurements use Iperf3. Every measurement took $1 \mathrm{~min}$ and was executed 100 times (300 times altogether).

\begin{tabular}{|c|c|c|c|c|c|}
\hline & $\begin{array}{c}\text { Average } \\
\text { (Gbps) }\end{array}$ & STDEV & MIN & MAX & Median \\
\hline VM-VM & 5.21 & 0.65 & 4.15 & 6.43 & 5.17 \\
\hline $\begin{array}{c}\text { Docker- } \\
\text { Docker }\end{array}$ & 5.32 & 0.66 & 4.22 & 6.50 & 5.23 \\
\hline $\begin{array}{c}\text { POD- } \\
\text { POD }\end{array}$ & 5.18 & 0.76 & 4.17 & 6.54 & 4.92 \\
\hline
\end{tabular}

Table 3. - Numerical results of User Plane measurements

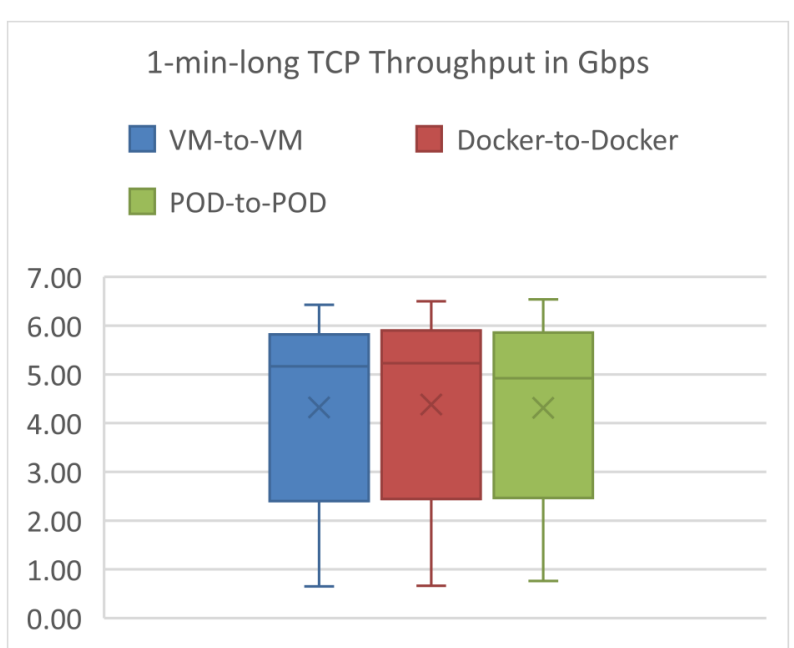

Figure 21- 1-min-long TCP throughput in case of IPv6-inIPv6 tunneling

Results: there is no significant difference between the three environments from the TCP throughput point of view when using IPv6-in-IPv6 tunneling (Table 3, Figure 21). However, the standard deviation of TCP throughput is increased when using Kubernetes Pods.

\section{E Measurements conclusion in overall}

The actual cost can be seen only on the control plane part of PMIPv6 when containerization is used. However, the standard deviation of the results is increased both in the control and user plane too. Containerization add more latency to control plane. This is the cost we have to pay when using containers and pods. However, we believe that the benefits of using microservices and cloud technologies together with their added features (e.g.: scaling, failover, software decoupling, complexity management, automatic deployment, easier upgradability, lower TCO etc. ) can and will easily overcome this minimal performance degradation.

\section{Conclusion}

In this paper, we presented a possible way how PMIPv6 can evolve into the "cloud". We proposed a containerized architecture on the top of Kubernetes for PMIPv6. The newly introduced CN-PMIPv6 
keeps the compatibility of IP-based mobility management standards; meanwhile, packet processing is handled in Kubernetes PODs. PMIPv6 has acquired new capabilities like scaling, which is essential in the cloud. With some measurements, we showed that containerization does not add very significant performance degradation for PMIPv6. At the end of this work phase, we think, PMIPv6 can become a CNF in the new telecommunication era.

\section{Future Work}

Our priority work is to provide a detailed design of Kubernetes Network Service while examining the usability of Network Service Mesh [37], Multus [38], DANM[39] and similar solutions in this context (e.g., decreasing the number of NATs). MAG can have a local breakout, which is also an exciting research topic on how to do it with CN-PMIPv6. Performance measures should take place between PMIPv6 and CN-PMIPv6, highlighting inter-MAG handovers. Furthermore, researching control flow between CPS and DPS has tremendous importance as well.

\section{ACKNOWLEDGMENTS}

The research reported in this paper was supported by the Higher Education Excellence Program in the frame of Artificial Intelligence research area of Budapest University of Technology and Economics (BME FIKP-MI/FM) and by the Center for University-Industry Cooperation (FIEK_16-1-2016-0007)

\section{REFERENCES}

[1] S. Gundavelli (Ed.), K. Leung, V. Devarapalli, K. Chowdhury, and B. Patil, Proxy Mobile IPv6. Fremont, CA, USA: RFC Editor, 2008. doi: 10.17487/RFC5213.

[2] H. Al-Hashimi, K. A. Bakar, and K. Ghafoor, 'Inter-domain Proxy Mobile IPv6 based Vehicular Network', Netw. Protoc. Algorithms, vol. 2, pp. 1-15, 2010.

[3] H. N. Hussain, K. A. Bakar, and S. Salleh, 'A Novel Intra-Domain Continues Handover Solution for Inter-Domain Pmipv6 Based Vehicular Network', International fournal of Advanced Computer Science and Applications, vol. 2, no. 12, 2011, doi: 10.14569/IJACSA.2011.021202.

[4] L. Le and M. Liebsch, 'Preliminary Binding: An Extension to Proxy Mobile IPv6 for Inter-Technology Handover', in 2009 IEEE Wireless Communication and Networking Conference, 2009, pp. 1-6. doi: 10.1109/WCNC.2009.4917918.

[5] R. Mijumbi, J. Serrat, J. Gorricho, N. Bouten, F. D. Turck, and R. Boutaba, 'Network Function Virtualization: State-of-the-Art and Research Challenges', IEEE Communications Surveys Tutorials, vol. 18, no. 1, pp. 236-262, 2016, doi: 10.1109/COMST.2015.2477041.

[6] N. McKeown et al., 'OpenFlow: Enabling Innovation in Campus Networks', SIGCOMM Comput. Commun. Rev., vol. 38, no. 2, pp. 69-74, Mar. 2008, doi: $10.1145 / 1355734.1355746$

[7] B. Han, V. Gopalakrishnan, L. Ji, and S. Lee, 'Network function virtualization: Challenges and opportunities for innovations', IEEE Communications Magazine, vol. 53, no. 2, pp. 90-97, 2015, doi: 10.1109/MCOM.2015.7045396.

[8] 'ETSI Multi-access Edge Computing'. https://www.etsi.org/technologies/multi-access-edge-computing (accessed Mar. 12, 2021).

[9] 'Openstack cloud platform official webpage'. https://www.openstack.org (accessed Mar. 12, 2021)

[10] 'Kubernetes container orchestration platform'. https://kubernetes.io/ (accessed Mar. 12, 2021)

[11] 'VMWARE Cloud Platform'. https://www.vmware.com/ (accessed Mar. 12, 2021).

[12] 'Google Cloud Platform'. https://cloud.google.com/ (accessed Mar. 12, 2021).

[13] Á. Leiter, L. Bokor, and I. Kispál, 'An Evolution of Mobile IPv6 to the Cloud', in Proceedings of the 18th ACM Symposium on Mobility Management and Wireless Access, New York, NY, USA, 2020, pp. 137-141. doi: 10.1145/3416012.3424633

[14] S. Kim, H. Choi, P. Park, S. Min, and Y. Han, 'OpenFlow-based Proxy mobile IPv6 over software defined network (SDN)', in 2014 IEEE 11th Consumer
Communications and Networking Conference (CCNC), Jan. 2014, pp. 119-125. doi: 10.1109/CCNC.2014.6866558.

[15] S. M. Raza, D. S. Kim, D. Shin, and H. Choo, 'Leveraging proxy mobile IPv6 with SDN', Journal of Communications and Networks, vol. 18, no. 3, Art. no. 3 Jun. 2016, doi: 10.1109/JCN.2016.000061.

[16] K. Hee Lee, 'Mobility Management Framework in Software Defined Networks', International fournal of Software Engineering and Its Applications, vol. 8, no. 8, pp. 1-10

[17] Y. Wang and J. Bi, 'A solution for IP mobility support in software defined networks', in 2014 23rd International Conference on Computer Communication and Networks (ICCCN), 2014, pp. 1-8. doi: 10.1109/ICCCN.2014.6911783.

[18] S. Chourasia and K. M. Sivalingam, 'SDN based Evolved Packet Core architecture for efficient user mobility support', in Proceedings of the 2015 1st IEEE Conference on Network Softwarization (NetSoft), Apr. 2015, pp. 1-5. doi: 10.1109/NETSOFT.2015.7116148.

[19] 'Service component of Kubernetes' https://kubernetes.io/docs/concepts/services-networking/service/ (accessed Mar. 12, 2021).

[20] 'Deployment element of Kubernetes'. https://kubernetes.io/docs/concepts/workloads/controllers/deployment/ (accessed Mar. 12, 2021)

[21] 'ETSI Management and Orchestration Architecture (MANO)'. https://www.etsi.org/deliver/etsi_gs/NFVMAN/001_099/001/01.01.01_60/gs_NFV-MAN001v010101p.pdf

[22] 'Open Network Automation Platform (ONAP)'. https://www.onap.org/ (accessed Jul. 05, 2021).

[23] 'ONAP and ETSI Cointainer support proposals' https://wiki.onap.org/display/DW/ETSI-Aligned+CNF+Support+-+Honolulu (accessed Mar. 15, 2021)

[24] 'Kubernetes on the Edge'. https://k3s.io/ (accessed Mar. 12, 2021).

[25] W. Kiess, E. Lang, K. Hoffmann, H.-J. Morper, and J. Varga, 'Ultra-reliable low latency services: $5 \mathrm{G}$ architecture and operational alternatives with cost analysis', in 2019 IEEE Wireless Communications and Networking Conference (WCNC), 2019, pp. 1-8. doi: 10.1109/WCNC.2019.8885604.

[26] W. Kiess, M. R. Sama, J. Varga, J. Prade, H.-J. Morper, and K. Hoffmann, '5G via evolved packet core slices: Costs and technology of early deployments', in 2017 IEEE 28th Annual International Symposium on Personal, Indoor, and Mobile Radio Communications (PIMRC), 2017, pp. 1-7. doi 10.1109/PIMRC.2017.8292691.

[27] J. Varga, A. Hilt, J. Bíró, C. Rotter, and G. Jaro, 'Reducing operational costs of ultra-reliable low latency services in 5G', Infocommunications fournal, vol. X, pp. 37-45, 2018, doi: 10.36244/ICJ.2018.4.6.

[28] 3GPP TS 23.402, Architecture enhancements for non-3GPP accesses", Rel. 11.

[29] '3GPP TS 29.275: Proxy Mobile IPv6 (PMIPv6) based Mobility and Tunnelling protocols; $\quad$ Stage 3 '. https://portal.3gpp.org/desktopmodules/Specifications/SpecificationDetails.a spx?specificationId $=1693$

[30] 'Cinder: storage component of Openstack'. https://wiki.openstack.org/wiki/Cinder (accessed Mar. 12, 2021).

[31] 'Swift: Object storage component of Openstack'. https://wiki.openstack.org/wiki/Swift (accessed Mar. 12, 2021).

[32] 'HEAT component of Openstack'. https://wiki.openstack.org/wiki/Heat (accessed Jul. 05, 2021).

[33] 'OASIS Topology and Orchestration Specification for Cloud Applications (TOSCA) TC'. https://www.oasisopen.org/committees/tc_home.php?wg_abbrev=tosca (accessed Mar. 17, 2021).

[34] 'ONAP Optimisation Framework'. https://wiki.onap.org/display/DW/5G+$+\mathrm{OOF}+(\mathrm{ONAP}+\mathrm{Optimization}+$ Framework $)+$ and $+\mathrm{PCI}+(\mathrm{Physical}+\mathrm{Cell}+\mathrm{ID})+\mathrm{Op}$ timization (accessed Mar. 15, 2021).

[35] 'Kubernetes Federation'. https://github.com/kubernetes-sigs/kubefed (accessed Mar. 12, 2021)

[36] C. Bernardos (Ed.), 'Proxy Mobile IPv6 Extensions to Support Flow Mobility'. RFC Editor, Fremont, CA, USA, May 2016. [Online]. Available: https://www.rfc-editor.org/rfc/rfc7864.txt

[37] 'Network Service Mesh'. https://networkservicemesh.io/ (accessed Mar. 12 , 2021).

[38] 'Multus Kubernetes meta-CNI'. https://github.com/k8snetworkplumbingwg/multus-cni (accessed Jun. 15, 2021).

[39] 'DANM: a Kubernetes container networking'. https://github.com/nokia/danm (accessed Feb. 12, 2021) 